\title{
Construcción de la escala de motivación de logro para el estudio
}

\section{Construction of the achievement motivation scale for the study}

\author{
Giannina Sovero Lazo ${ }^{1 *}$, Gaspar Orellana Méndez ${ }^{1}$ \\ ${ }^{1}$ Universidad Nacional del Centro del Perú, Huancayo
}

\section{RESUMEN}

Se realizó la construcción y el análisis psicométrico de una Escala de Motivación de Logro para el Estudio (EMLPE), en base a los constructos teóricos de Weiner (1989) sobre las motivaciones y atribuciones que realizan las personas para explicar las causas de las conductas y acontecimientos de la realidad. La muestra fue de 517 postulantes a la Universidad Continental, varones y mujeres de 18 a 49 años de edad, residentes en los departamentos de Junín, Lima, Arequipa y la provincia constitucional del Callao, entre otros; para ello se aplicó la escala inicial de 20 ítems vía internet y luego del análisis de confiabilidad con el alfa de Crombach $(0,73)$ y la validez empírica ítem total a través de la $r$ de Pearson corregida con valores mayores a 0,20, quedaron 14 ítems que fueron agrupadas en 3 factores mediante del análisis factorial de rotación varimax. Los resultados obtenidos muestran que pese a que la escala MLPE no pasó por el juicio de expertos, cuenta con adecuadas propiedades psicométricas de confiabilidad y validez. La escala presenta una buena consistencia interna, ya que el alfa de Crombach excede del criterio mínimo recomendado de 0,70 . Los 14 ítems definitivos presentan una $r$ de Pearson Corregida con valores iguales o mayores a 0,20 , lo cual confirma la validez empírica ítem test de la escala. El análisis factorial con rotación Varimax de la escala y la consistencia interna de la misma reflejan que los 14 ítems son adecuados indicadores del constructo con cargas factoriales mayores a 0,40.

Palabras claves: Validez, confiabilidad, análisis factorial, motivación de logro.

\section{ABSTRACT}

The construction and the psychometric analysis of a Scale of Motivation of Achievement for the study (EMLPE) was carried out, based on the theoretical constructs of Weiner (1989) on the motivations and atributions that people make to explain the causes of behaviors and events of reality. The sample was of 517 applicants to the Universidad Continental, men and women from 18 to 49 years of age, residing in the departments of Junín, Lima, Arequipa and the constitutional province of Callao, among others; for this the initial scale of 20 items was applied via internet and after the analysis of reliability with the Crombach alpha $(0,73)$ and the empirical validity of the total ítem through the corrected Pearson's $r$ with values greater than 0,20 , there were 14 items that were grouped into 3 factors throught the factors analysis of varimax rotation. The results obtained show that although expert judgment, it has adequate psychometric properties of reliability and validity. The scale has good internal consistency, since Crombach's alpha exceeds the minium recommended criterion of 0,70 . The 14 definitive ítems present a corrected Pearson $r$ with values esqual to or greater than 0,20 , which confirms the empirical validity of the scale test ítem. The factor analysis with Varimax rotation of the scale and the internal consistency of the scale reflect that the 14 items are adequate indicators of the construct with factor loads greater than 0,40 .

Keywords: Validity, reliability, factor analysis, achievement motivation.

Historial del artículo:

Recibido, 12 de abril de 2016; aceptado, 10 de enero de 2017; disponible en línea, 25 de junio de 2017

* Psicóloga, docente de la Universidad Continental.

Correo: jsovero@continental.edu.pe 


\section{INTRODUCCIÓN}

\section{La teoría de la Atribución de Weiner}

La teoría de la atribución explica como interpretan las personas las causas de las conductas y las consecuencias que tienen los acontecimientos propios y de otras personas.

La interpretación que realizan las personas de los hechos está guiada por sus propias creencias, valores y sentimientos y mediante la interpretación se atribuyen las causas y los resultados de las conductas y acontecimientos a causas que pueden ser externas o internas, controlables o incontrolables.

Las atribuciones se relacionan con las motivaciones de las personas y llegan a influir en las conductas, estrategias y relaciones que establecen con el mundo en general y en la vida cotidiana en particular, así como en los contextos de aprendizaje y en los contextos laborales.

Las atribuciones negativas influyen negativamente en las conductas que realizan las personas y en las que no realizan por temor a fracasar y las atribuciones positivas influyen positivamente en las conductas de éxito y en las consecuencias positivas.

En el contexto escolar y de aprendizaje es importante favorecer y promover atribuciones positivas para impulsar y estimular el aprendizaje, para motivar al alumno a aprender y a controlar sus éxitos y sus fracasos.

Weiner ha propuesto tres dimensiones para explicar las atribuciones que hacen las personas: Localización, la estabilidad y la capacidad de control.

Estas 3 dimensiones son esenciales para entender según Weiner (1989) las motivaciones y atribuciones que realizan las personas para explicar las causas de las conductas y acontecimientos de la realidad.

La percepción sobre el funcionamiento del mundo guía las atribuciones que se realizan y la gente explica sus conductas y la de los demás en función de las atribuciones que hacen sobre lo que sucede y las causas que producen esos sucesos.

Las tres dimensiones propuestas por Weiner explican cómo se realizan las atribuciones, veamos:

\section{Localización}

La dimensión denominada: localización, de Weiner, es la que otros autores denominan Locus de control.

Considera dos clases de atribuciones de localización: a) Interna: Las causas de los acontecimientos se interpretan preferentemente como causas internas, por ejemplo: He estudiado poco este examen y lo he suspendido

b) Externa: Las causas de los acontecimientos se interpretan preferentemente en función de sucesos externos a nosotros: No he aprobado el examen porque no he tenido suerte y siempre lo ponen difícil.

\section{Estabilidad}

La dimensión de estabilidad hace referencia a dos factores:

a) Estables: que funcionan como causas de los acontecimientos son interpretados como factores que no puede cambiar el sujeto ya que no dependen de él, por ejemplo: suspendo porque soy poco inteligente y la inteligencia es innata, este tipo de atribución es la que hace que los sujetos piensen que por mucho que se esfuercen terminarán suspendiendo por que no son inteligentes. Los factores estables se consideran que son inmodificables y que no cambiaran porque no dependen de nosotros, son causas externas con poca probabilidad de cambiar y que nos influyen positiva o negativamente.

b) Inestables: son causas que pueden cambiar, por ejemplo el día que realizó el examen estaba muy cansado y es la causa de que lo suspendiera. Estar cansado es un factor inestable ya que puede modificarse.

\section{La capacidad de control}

La dimensión de la capacidad de control es la capacidad de cambiar las causas que producen los acontecimientos, hay factores controlables y factores incontrolables:

a) Controlables: son causas que el sujeto puede modificar, controlar, por ejemplo si considera que el examen lo suspendió porque estaba cansado por haber salido de fiesta por la noche y regresar muy tarde, es un factor que puede controlar y cambiar.

b) Incontrolables: son causas que el sujeto considera que no puede controlar, ha suspendido el examen porque justo en ese momento le entró fiebre muy alta y eso le impidió realizarlo correctamente.

La posibilidad de controlar las causas es lo que define a esta dimensión, y es parecida al concepto de Autodeterminación propuesto por Decy y Ryan (1987) este concepto hace referencia a la creencia que tienen las personas de poder controlar las conductas y el ambiente. La capacidad de control, supone que las personas se valoran positivamente y creen ser capaces de controlar los sucesos, en general. 
Tienen sentido de:

- Autoeficacia, auto competencia.

- Establecen asociaciones entre las conductas y las respuestas que creen controlar.

En función de estas dos variables: auto competencia y asociación entre conducta y respuesta, valoran las posibilidades de los acontecimientos para saber si pueden controlarlos o no. Por ejemplo, si creen que el esfuerzo depende de las personas y es una variable importante para el deporte de natación, pero consideran que no tienen habilidades para ese deporte, entonces considerarán que no pueden controlar las respuestas de éxito en ese deporte.

La teoría de las atribuciones es una teoría que se basa en las creencias de las personas, y las tres dimensiones que propuso Weiner son de gran utilidad para ayudar a las personas y a los estudiantes a valorar sus propias creencias en relación al éxito y fracaso de sus conductas, y de su disposición a realizar o no esfuerzos para conseguir beneficios que dependen más de las creencias que de la realidad tal como el sujeto la valora. Los programas y el asesoramiento educativo, basados en las 3 dimensiones propuestas por Weimer, relacionadas con las creencias de los alumnos y su relación con el aprendizaje, éxito y fracaso, han demostrado ser eficaces para ayudar a los alumnos a valorar adecuadamente los factores que favorecen el aprendizaje y su posibilidad de modificar el éxito y el fracaso escolar.

Las atribuciones que hacemos sobre las conductas y acontecimientos, sobre los hechos que ocurren en la realidad, dependen de nuestras creencias y las creencias pueden modificarse para afrontar la realidad, escolar, profesional, laboral y personal de diferentes maneras, y esto permite que la teoría de las atribuciones pueda aplicarse como herramienta de cambio de pensamientos tanto en educción como en terapia constructiva, conductual-cognitiva.

La conducta futura en estas teorías de atribuciones, no dependen de factores externos, depende de la atribución que realicemos sobre los hechos, las causas y nuestra capacidad para determinar el comportamiento que tenemos y tienen otros.

La teoría de la atribución explica cómo interpretan las personas las causas de las conductas y las consecuencias que tienen. Weiner y las 3 dimensiones que inciden en los comportamientos, es una de las teorías que más ha influido en los cambios y en las motivaciones aplicadas a la realidad personal y escolar.

Validez. La validez es un concepto que tiene muchas facetas. Su establecimiento se enmarca dentro de un proceso. En una primera aproximación, puede significar "el grado en que una prueba mide lo que intenta medir". En todo proceso de validación, la validez de un test se expresa generalmente por medio de un coeficiente de correlación entre sus puntajes y los llamados criterios y, precisamente, la correlación entre los puntajes del test y las medidas de criterio se denomina coeficiente de validez, cuyo valor debe ser igual o mayor a 0.20 para que el ítem sea considerado válido.

Confiabilidad. Con el alfa da Crombach se llevan a cabo todas las posibles relaciones de mitades divididas, cuyo valor nunca debe ser menor de 0,70 para ser válido (Kline, 1993, referido por Clark-Carter, 2002).

El modelo de Análisis Factorial (AF) propiamente tal, según U. Kramp, (2008) incluye una serie de técnicas estadísticas de análisis multivariante diseñadas para: a) explorar la estructura subyacente o dimensiones básicas de un conjunto amplio de variables y b) para confirmar, a partir de un número amplio de variables observadas, un conjunto menor de constructos o variables, denominados factores o rasgos latentes. Éstos últimos son inferidos a partir de la puntuación observada o empírica $(Y)$ obtenida por el sujeto tras contestar uno o varios ítems que dan lugar a la escala utilizada para evaluar un constructo psicológico $(\eta)$ en particular. Aunque en todos los casos se aplica el mismo modelo general de $A F$, cuando éste se refiere exclusivamente al análisis de los ítems que componen una escala (p. ei., análisis de la capacidad discriminativa, dificultad de cada ítem, etc.), suele reservarse el nombre de Análisis Factorial de los Ítems (AFI).

Idealmente, las cargas factoriales en los análisis exploratorios deben ser iguales o mayores a .40 para ser considerados como parte de un factor (Field, 2005, referido por Matos, 2009)

\section{Antecedentes}

Núñez, Martin-Albo y Navarro (2005) adaptaron al español la Escala de Échele de Motivation en Education (EME) de Vellerand, Blais, Briere y Pelletier (1989) en 636 universitarios de la Universidad de 'Las Palmas de Gran Canaria. La escala se construyó originalmente bajo la teoría de la autodeterminación y lograron darle confiabilidad, ratificaron los siete factores de la prueba a través del análisis factorial confirmatorio y dieron validez de constructo a través de las correlaciones entre las 7 subescalas y hallaron diferencias significativas a favor de las mujeres.

L. Matos (2009), realizo una investigación psicométrica que tuvo como objetivo adaptar y validar dos cuestionarios: Autorregulación del Aprendizaje y Clima de Aprendizaje. La muestra estuvo compuesta por 369 estudiantes universitarios de una universidad privada de Lima con una edad promedio de 19,30 años (DE $=2,49)$. En ambos casos, se analizó la validez de 
constructo a través de análisis factoriales exploratorios con extracción de componentes principales, análisis factoriales confirmatorios y mediante la validez convergente y divergente de ambos cuestionarios. La validez de contenido se investigó a través del criterio de jueces expertos, mientras que la validez predictiva se llevó a cabo a través de correlaciones y regresiones entre las variables estudiadas (clima y regulación del aprendizaje) y el rendimiento académico de los alumnos. La confiabilidad se verificó a través del método de consistencia interna (Alfa de Cronbach).

El objetivo fue construir, validar, y elaborar baremos de una escala para medir la motivación de logro para el estudio, bajo el soporte teórico de la categoría de la atribución planteada por Weiner (1989), en postulantes a la Universidad Continental en el periodo 2013 III.

\section{MATERIAL Y MÉTODOS}

El estudio se realizó en 517 postulantes a la Universidad Continental que se presentaron al examen de admisión en el 2013, de los cuales 353 fueron varones $(68,3$ $\%)$ y 164 mujeres $(31,7 \%)$, residentes en Lima $(44,5$ $\%)$, Junín (35,4 \%), Arequipa (8,9 \%), Callao (3,5\%) y otros departamentos del Perú $(7,7 \%)$, de 18 a 49 años y con edad promedio de 30,78 y desviación estándar de 6,91.

El instrumento inicialmente propuesto fue de un cuestionario que constaba de 20 ítems, agrupados en tres factores o dimensiones: Localización (8 ítems), Estabilidad (6 ítems) y Control (6 ítems), 14 reactivos positivos y 6 negativos (tabla 1).

Las alternativas de respuestas se organizaron en una escala tipo Likert, que va de nunca (1), a veces (2) a siempre (3), y cuando se trata de ítems negativos se invierte la puntuación.

Respecto a los procedimientos, a partir de la teoría de la atribución de Weiner (1989) se elaboró la matriz lógica de la escala, se hizo la definición operacional de la variable y de las tres dimensiones a partir de esta teoría. Luego se construyó la escala con 20 ítems y se insertó en una plataforma virtual para que sea contestada por los postulantes a las carreras profesionales virtuales, vía internet entre el 25 de julio al 19 de noviembre, en un tiempo libre de resolución de 2 a 14 minutos. Los datos fueron almacenados en el programa Excel, se revisaron las respuestas y luego

Tabla 1. Matriz lógica de la escala de motivación de logro para el estudio.

\begin{tabular}{|c|c|c|c|}
\hline Variable & Dimensiones & Indicadores & Reactivos \\
\hline \multirow{4}{*}{$\begin{array}{l}\text { Motivación de } \\
\text { logro para el } \\
\text { estudio } \\
\text { (Weiner, 1989) }\end{array}$} & \multirow{3}{*}{$\begin{array}{l}\text { Localización } \\
\text { Localización interna } \\
\text { en oposición a la } \\
\text { atribución externa: }\end{array}$} & 2. Sentirse ante los demás & 2. Con frecuencia me siento menos que los demás (-) \\
\hline & & 3. Autoevaluación de cualidades & 3. Creo que tengo más cualidades positivas que negativas \\
\hline & & $\begin{array}{l}\text { 5. Responsabilidad ante decisiones } \\
\text { tomadas }\end{array}$ & $\begin{array}{l}\text { 5. Estoy consciente de que todas mis decisiones tienen } \\
\text { consecuencias y me hago responsable de ellas. }\end{array}$ \\
\hline & \multirow{3}{*}{$\begin{array}{l}\text { Las causas de los } \\
\text { acontecimientos } \\
\text { se interpretan }\end{array}$} & 8. Afección ante la desaprobación. & 8. Me afecta que las personas me desaprueben \\
\hline Proceso & & 10. Emocion al tiempo y los deseos & 10. Me asusta perder el tiempo y no alcanzar lo que deseo (-) \\
\hline de valorar & & 14. Satisfacion consigo mismo & 14. Me siento satisfecho(a) conmigo mismo \\
\hline las propias & \multirow{2}{*}{$\begin{array}{l}\text { preferentemente } \\
\text { como causas } \\
\text { internas. }\end{array}$} & 17. Reconocer habilidades propias & 17. Recono \\
\hline $\begin{array}{l}\text { creencias en } \\
\text { relación al éxito }\end{array}$ & & 18. Satisfacción con la vida que lleva & 18. Siento satisfacción con la vida que estoy llevando \\
\hline \multirow{2}{*}{$\begin{array}{l}\text { y fracaso de sus } \\
\text { conductas, y de }\end{array}$} & \multirow{3}{*}{$\begin{array}{l}\text { Estabilidad } \\
\text { Factores estables vs } \\
\text { factores inestables: }\end{array}$} & 7. Calidad de las relaciones & 7. Las personas se relacionan conmigo por lo que soy no por \\
\hline & & & \\
\hline su disposición & & 9. Deseo de tener futuro exitoso & 9. Me angustia la idea de no tener un futuro exitoso(-) \\
\hline \multirow{3}{*}{$\begin{array}{l}\text { a realizar o } \\
\text { no esfuerzos } \\
\text { para conseguir }\end{array}$} & \multirow{3}{*}{$\begin{array}{l}\text { Los factores } \\
\text { estables no } \\
\text { cambiaran porque } \\
\text { no dependen de }\end{array}$} & 11. Búsqueda de admiración & 11. Me gustaría ser alguien admirable en la vida. \\
\hline & & $\begin{array}{l}\text { 13. Valoración de los trabajos } \\
\text { propios }\end{array}$ & $\begin{array}{l}\text { 13. Me parece que los trabajos de mis compañeros son } \\
\text { mejores que los míos (-) }\end{array}$ \\
\hline & & 15. Trascender en la vida & 15. No me import \\
\hline beneficios que & \multirow{3}{*}{$\begin{array}{l}\text { nosotros, y que nos } \\
\text { influyen positiva o } \\
\text { negativamente }\end{array}$} & 16. Ser considerado valioso en la & 16. Quie \\
\hline dependen más & & & \\
\hline de las creencias & & & \\
\hline \multirow{6}{*}{$\begin{array}{l}\text { que de la } \\
\text { realidad tal } \\
\text { como el sujeto la } \\
\text { valora. }\end{array}$} & \multirow{6}{*}{$\begin{array}{l}\text { La capacidad de } \\
\text { control } \\
\text { Controlables } \\
\text { en oposición a } \\
\text { incontrolables: } \\
\text { son causas que } \\
\text { el sujeto puede } \\
\text { modificar, manejar. }\end{array}$} & 1. Firmeza en la toma de decisiones & $\begin{array}{l}\text { 1. Cada vez que tomo una decisión me mantengo firme en } \\
\text { ella. }\end{array}$ \\
\hline & & 4. Control de sí mismo & 4. En general tengo buen control sobre mí mismo \\
\hline & & 6. Temor al fracaso & 6. Evitaría el fracaso a toda costa \\
\hline & & $\begin{array}{l}\text { 12. Vida significativa y trascendente } \\
\text { 19. Hacer actividades normales }\end{array}$ & $\begin{array}{l}\text { 12. Me inquieta tener una vida sin significado y } \sin \\
\text { trascendencia (-) }\end{array}$ \\
\hline & & & $\begin{array}{l}\text { 19. Soy una persona a la que le gusta caminar, leer o hacer } \\
\text { otras actividades recreativas }\end{array}$ \\
\hline & & 20. Constancia en el logro de metas & 20. Tengo la constancia suficiente para lograr mis metas \\
\hline
\end{tabular}


se trasladaron al programa SPSS versión 15 para ser procesados estadísticamente.

Para la confiabilidad se aplicó el Alfa de Crombach y para la validez la $r$ de Pearson Corregida, a partir de los resultados obtenidos se seleccionaron como válidos 14 ítems, los cuales se agruparon en tres factores según los resultados del análisis factorial de los ítems, Confirmatorio, Exploratorio y rotación Varimax, que determinó el instrumento definitivo. Finalmente, dado que no se encontraron diferencias significativas según sexo y edad, se construyeron baremos, escala y percentil, para la variable y sus dimensiones. En el caso de la escala se convirtió la puntuación directa a puntuación estándar, por medio de la suma y resta de una desviación estándar al puntaje promedio, obteniendo de esta forma tres niveles, alto, medio y bajo. Y en caso de percentil a partir de las frecuencias directas y teniendo en cuenta los puntajes obtenidos en la escala se elaboraron percentiles en amplitud de 10 para toda la escala y sus tres dimensiones.

\section{RESULTADOS}

\section{Validez y confiabilidad}

El Alfa de Crombach obtenido fue de 0,677, valor no aceptado para considerarse que las puntuaciones presenten estabilidad. La tabla 2 presenta los puntajes promedios y la desviación típica, la media más alta se dio en el ítem 20: Tengo la constancia suficiente para lograr mis metas y la más baja en el ítem 6: Evitaría el fracaso a toda costa, el resto de reactivos obtuvieron medias por encima del puntaje medio de 2 evidenciado niveles altos. La mayor dispersión de los datos se dio en el ítem 12: Me inquieta tener una vida sin significado y sin trascendencia y la menor dispersión en el ítem 5: Estoy consciente de que todas mis decisiones tienen consecuencias y me hago responsable de ellas, la mayoría de ítems presentan dispersión alta dado que el menor número de sujetos marcaron la alternativa nunca(1), concentrándose sus respuestas mayoritariamente en la alternativa a veces (2) y siempre (3), dado que como es un examen de admisión los sujetos tienden a marcar la respuesta que consideran más acertada.

También vemos en la misma tabla, que el análisis de validez empírica ítem test arroja valores de la r de Pearson Corregida iguales o mayores de 0.20 en la mayoría de los ítems, excepto en los ítems 5: Estoy consciente de que todas mis decisiones tienen consecuencias y me hago responsable de ellas, 6: Evitaría el fracaso a toda costa, 7: Las personas se relacionan conmigo por lo que soy no por lo que les doy, 15: No me importa morir sin trascender y 16: Quiero que me recuerden como un ser valioso para la sociedad. Por lo visto resultan validos 14 ítems, de los cuales 8 están redactados en sentido positivo y 6 en sentido negativo.

Tabla 2: Media, D.T. y $r$ de Pearson Corregida de los 20 ítems de la escala MLPE.

\begin{tabular}{|c|c|c|c|}
\hline Items motivacion de logro para el estudio & Media & D.T. & $\begin{array}{c}r \text { de } P \\
\text { corregida }\end{array}$ \\
\hline 1. Cada vez que tomo una decisión me mantengo firme en ella & 2,82 & 0,399 & 0,264 \\
\hline 2. Con frecuencia me siento menos que los demás (-) & 2,80 & 0,425 & 0,310 \\
\hline 3. Creo que tengo más cualidades positivas que negativas & 2,81 & 0,413 & 0,197 \\
\hline 4. En general tengo buen control sobre mí mismo & 2,76 & 0,452 & 0,332 \\
\hline $\begin{array}{l}\text { 5. Estoy consciente de que todas mis decisiones tienen consecuencias y me } \\
\text { hago responsable de ellas }\end{array}$ & 2,95 & 0,227 & 0,142 \\
\hline 6. Evitaria el fracaso a toda costa & 1,28 & 0,520 & 0,003 \\
\hline 7. Las personas se relacionan conmigo por lo que soy no por lo que les doy & 2,74 & 0,459 & 0,093 \\
\hline 8. Me afecta que las personas me desaprueben (-) & 2,28 & 0,595 & 0,370 \\
\hline 9. Me angustia la idea de no tener un futuro exitoso(-) & 2,13 & 0,761 & 0,518 \\
\hline 10. Me asusta perder el tiempo y no alcanzar lo que deseo (-) & 1,94 & 0,775 & 0,468 \\
\hline 11. Me gustaría ser alguien admirable en la vida & 2,88 & 0,379 & 0,014 \\
\hline 12. Me inquieta tener una vida sin significado y sin trascendencia (-) & 1,94 & 0,841 & 0,344 \\
\hline 13. Me parece que los trabajos de mis compañeros son mejores que los míos (-) & 2,45 & 0,517 & 0,242 \\
\hline 14. Me siento satisfecho (a) conmigo mismo & 2,73 & 0,497 & 0,438 \\
\hline 15. No me importa morir sin trascender (-) & 2,59 & 0,639 & 0,085 \\
\hline 16. Quiero que me recuerden como un ser valioso para la sociedad & 2,83 & 0,415 & 0,050 \\
\hline 17. Reconozco que soy hábil para muchas cosas & 2,90 & 0,325 & 0,200 \\
\hline 18. Siento satisfacción con la vida que estoy llevando & 2,66 & 0,539 & 0,372 \\
\hline $\begin{array}{l}\text { 19. Soy una persona a la que le gusta caminar, leer o hacer otras actividades } \\
\text { recreativas }\end{array}$ & 2,78 & 0,435 & 0,239 \\
\hline 20. Tengo la constancia suficiente para lograr mis metas & 2,92 & 0,64 & 0,305 \\
\hline
\end{tabular}


Ahora con los 14 ítems validos procesamos el Alfa de Crombach y resulta el valor de 0,727 , lo cual nos permite afirmar que el instrumento es confiable, es decir las puntuaciones presentan estabilidad. La tabla 3 presenta los valores de la media, la desviación típica y la $r$ de Pearson Corregida. Como es obvio las puntuaciones medias y desviaciones típicas

\section{Análisis factorial de los ítems}

Una vez obtenida la validez de 14 ítems, se procedió a determinar la estructura interna del cuestionario en las dimensiones o factores que lo conformarían, para lo cual se realizó el análisis factorial de los ítems.

Tabla 3: Media, D.T. y $r$ de Pearson Corregida de los 14 ítems de la escala MLPE.

\begin{tabular}{lccc}
\hline \multicolumn{1}{c}{ Items motivacion de logro para el estudio } & Media & D.T. & $\begin{array}{c}\text { r de } P \\
\text { corregida }\end{array}$ \\
\hline 1. Cada vez que tomo una decisión me mantengo firme en ella & 2,82 & 0,399 & 0,249 \\
2. Con frecuencia me siento menos que los demás (-) & 2,80 & 0,425 & 0,331 \\
3. Creo que tengo más cualidades positivas que negativas & 2,81 & 0,413 & 0,199 \\
4. En general tengo buen control sobre mi mismo & 2,76 & 0,452 & 0,341 \\
5. Me afecta que las personas me desaprueben (-) & 2,28 & 0,595 & 0,382 \\
6. Me angustia la idea de no tener un futuro exitoso(-) & 2,13 & 0,761 & 0,550 \\
7. Me asusta perder el tiempo y no alcanzar lo que deseo (-) & 1,94 & 0,775 & 0,500 \\
8. Me inquieta tener una vida sin significado y sin trascendencia (-) & 1,94 & 0,841 & 0,369 \\
9. Me parece que los trabajos de mis compañeros son mejores que los míos (-) & 2,45 & 0,517 & 0,250 \\
10. Me siento satisfecho (a) conmigo mismo & 2,73 & 0,497 & 0,451 \\
11. Reconozco que soy hábil para muchas cosas & 2,90 & 0,325 & 0,202 \\
12. Siento satisfacción con la vida que estoy llevando & 2,66 & 0,539 & 0,399 \\
13. Soy una persona a la que le gusta caminar, leer o hacer otras actividades & 2,78 & 0,435 & 0,229 \\
recreativas & & & \\
14. Tengo la constancia suficiente para lograr mis metas & 2,92 & 0,264 & 0,281 \\
\hline
\end{tabular}

Tabla 4: Análisis factorial confirmatorio de los 14 ítems de la escala MLPE.

\begin{tabular}{lrrr}
\hline \multicolumn{1}{c}{ Items motivacion de logro para el estudio } & \multicolumn{3}{c}{ Componente } \\
\cline { 2 - 4 } & $\mathbf{1}$ & \multicolumn{1}{c}{$\mathbf{2}$} \\
\hline 1. Cada vez que tomo una decisión me mantengo firme en ella & 0,378 & 0,270 & 0,232 \\
2. Con frecuencia me siento menos que los demás (-) & 0,464 & 0,066 & 0,492 \\
3. Creo que tengo más cualidades positivas que negativas & 0,292 & 0,314 & $-0,322$ \\
4. En general tengo buen control sobre mí mismo & 0,482 & 0,259 & 0,101 \\
5. Me afecta que las personas me desaprueben (-) & 0,515 & $-0,200$ & 0,388 \\
6. Me angustia la idea de no tener un futuro exitoso(-) & 0,665 & $-0,425$ & 0,072 \\
7. Me asusta perder el tiempo y no alcanzar lo que deseo (-) & 0,611 & $-0,462$ & $-0,050$ \\
8. Me inquieta tener una vida sin significado y sin trascendencia (-) & 0,493 & $-0,498$ & $-0,148$ \\
9. Me parece que los trabajos de mis compañeros son mejores que los míos (-) & 0,360 & $-0,031$ & 0,167 \\
10. Me siento satisfecho (a) conmigo mismo & 0,605 & 0,066 & $-0,401$ \\
11. Reconozco que soy hábil para muchas cosas & 0,310 & 0,404 & $-0,164$ \\
12. Siento satisfacción con la vida que estoy llevando & 0,548 & 0,107 & $-0,589$ \\
13. Soy una persona a la que le gusta caminar, leer o hacer otras actividades & 0,365 & 0,512 & 0,082 \\
recreativas & & & \\
14. Tengo la constancia suficiente para lograr mis metas & 0,409 & 0,405 & 0,272 \\
\hline
\end{tabular}

Método de extracción: Análisis de componentes principales.

a 3 componentes extraídos

se mantienen, pero varían las de la $r$ de Pearson Corregida, pero todas ellas con valores iguales o mayores a 0,20, siendo las más baja la del ítem 3: Creo que tengo más cualidades positivas que negativas $(r=0,199)$ y la más alta la del ítem 6: Me angustia la idea de no tener un futuro exitoso $(r=0,650)$.
El análisis factorial confirmatorio (AFC). En primer lugar, se trató de comprobar si la estructura interna de la prueba se ratificaba en tres componentes tal como lo plantea la teoría, por lo que se realizó el AFC. El resultado del análisis de componentes principales resultó con tres componentes con autovalores (Eigen) mayores a 1, los cuales explicaron un $42,529 \%$ del 
total de la varianza. El autovalor (Eigen) del primer componente fue de 3.190 y explicó el $22,788 \%$ de la varianza. El segundo componente obtuvo un autovalor de 1,531 explicando el 10,934\% de la varianza, mientras que el tercer componente obtuvo un autovalor de 1,233 y explicó un $8,807 \%$ del total de la varianza.

Según la tabla 4, las cargas factoriales alcanzaron valores entre 0,332 y 0,665 y todas fueron significativas, de tal manera que la estructura de 3 factores comprenderían los ítems 1, 4, 5, 6, 7, 9, 10 y 14 para el primer factor (8 ítems), los ítems 8,11 y 13 para el segundo factor ( 3 ítems) y los ítems 2,3 y 12 para el tercer factor (3 ítems).

En vista que estos resultados rompe la proporcionalidad de la cantidad de ítems por factor (8-3-3) y los enunciados de los ítems no concuerdan con las cuenta, como ya se indicó, que dichos análisis eran solo de carácter exploratorio.

Está situación nos llevó a probar el análisis factorial con el Método de rotación: Normalización Varimax con Kaiser.

Análisis factorial de rotación Varimax. Vemos en la tabla cinco que este análisis divide a la escala en tres factores, con cargas factoriales entre 0,292 y 0,765 y solo el ítem 9 presenta una carga factorial menor de 0,40 . La proporción de ítems por factor es adecuada. Factor uno con cuatro ítems $(5,6,7$ y 8$)$, factor dos con seis ítems $(1,2,4,9,12$ y 14) y factor tres con cuatro ítems $(3,10,11$ y 12). El análisis del contenido de los ítems con la definición operacional de los factores ratifica este resultado, por lo que la matriz lógica definitiva se resume en el cuadro dos, donde el factor

Tabla 5. Análisis factorial exploratorio de los 14 ítems de la escala MLPE.

\begin{tabular}{lrrrr}
\hline \multicolumn{1}{c}{ Items motivacion de logro para el estudio } & \multicolumn{3}{c}{ Componente } \\
\cline { 2 - 5 } & 1 & 2 & \multicolumn{1}{c}{3} & \multicolumn{1}{c}{4} \\
\hline 1. Cada vez que tomo una decisión me mantengo firme en ella & 0,378 & 0,270 & 0,232 & $-0,037$ \\
2. Con frecuencia me siento menos que los demás (-) & 0,464 & 0,066 & 0,492 & 0,413 \\
3. Creo que tengo más cualidades positivas que negativas & 0,292 & 0,314 & $-0,322$ & $-0,270$ \\
4. En general tengo buen control sobre mi mismo & 0,482 & 0,259 & 0,101 & $-0,250$ \\
5. Me afecta que las personas me desaprueben (-) & 0,515 & $-0,200$ & 0,388 & $-0,071$ \\
6. Me angustia la idea de no tener un futuro exitoso(-) & 0,665 & $-0,425$ & 0,072 & $-0,184$ \\
7. Me asusta perder el tiempo y no alcanzar lo que deseo (-) & 0,611 & $-0,462$ & $-0,050$ & $-0,211$ \\
8. Me inquieta tener una vida sin significado y sin trascendencia (-) & 0,493 & $-0,498$ & $-0,148$ & $-0,129$ \\
9. Me parece que los trabajos de mis compañeros son mejores que los míos (-) & 0,360 & $-0,031$ & 0,167 & 0,633 \\
10. Me siento satisfecho (a) conmigo mismo & 0,605 & 0,066 & $-0,401$ & 0,188 \\
11. Reconozco que soy hábil para muchas cosas & 0,310 & 0,404 & $-0,164$ & 0,190 \\
12. Siento satisfacción con la vida que estoy llevando & 0,548 & 0,107 & $-0,589$ & 0,284 \\
13. Soy una persona a la que le gusta caminar, leer o hacer otras actividades & 0,365 & 0,512 & 0,082 & $-0,269$ \\
recreativas & & & & \\
14. Tengo la constancia suficiente para lograr mis metas & 0,409 & 0,405 & 0,272 & $-0,206$ \\
\hline Método de extracción: Análisis de componentes principales. a 4 componentes extraídos. & & &
\end{tabular}

definiciones operacionales de los factores, se procesó el análisis factorial exploratorio.

Análisis factorial exploratorio (AFE). Este análisis arrojo cuatro componentes con autovalores (Eigen) de $3,190,1,531,1,233,1,076$ explicando el $22,788 \%, 10,934 \%, 8,807 \%$ y $7,683 \%$ de la varianza total, respectivamente. Los cuatro componentes explicaron el 50,212\% del total de la varianza. Esta vez, los ítems alcanzaron cargas factoriales con valores entre 0,322 y 0,665 (véase la tabla 4). Ahora el primer factor tendría siete ítems, el segundo tres ítems, el tercero tres ítems y el cuarto un ítem. Sin embargo, sigue la desproporcionalidad de numero de ítems por factor y varios ítems contenían cargas factoriales altas en dos o tres componentes, y ello no permitió realizar un juicio definitivo acerca de la estructura factorial de la escala mediante estos análisis. Debe tenerse en uno es la dimensión Localización, el factor dos Control y el factor tres Estabilidad.

\section{Baremos}

Al no encontrar diferencias significativas en la motivación de logro para el estudio ni en sus dimensiones, según sexo y edad, se elaboraron escalas y percentiles únicos.

a) Escalas: La tabla 8 presenta tres niveles para la variable y sus tres dimensiones, considerando los respectivos puntajes directos para cada nivel.

b) Percentiles: La tabla 9 presenta los percentiles y sus puntuaciones directas correspondientes de la motivación de logro para el estudio y de sus dimensiones. 
Tabla 6: Análisis factorial de rotación Varimax de los 14 ítems de la escala MLPE.

\begin{tabular}{lrrr}
\hline \multicolumn{1}{c}{ Items motivacion de logro para el estudio } & \multicolumn{3}{c}{ Componente } \\
\cline { 2 - 4 } & \multicolumn{1}{c}{ 1 } & \multicolumn{1}{c}{3} \\
\hline 1.Cada vez que tomo una decisión me mantengo firme en ella & 0,061 & 0,501 & 0,121 \\
2. Con frecuencia me siento menos que los demás (-) & 0,270 & 0,611 & $-0,125$ \\
3. Creo que tengo más cualidades positivas que negativas & $-0,032$ & 0,117 & 0,522 \\
4. En general tengo buen control sobre mí mismo & 0,140 & 0,468 & 0,267 \\
5. Me afecta que las personas me desaprueben (-) & 0,499 & 0,433 & $-0,138$ \\
6. Me angustia la idea de no tener un futuro exitoso(-) & 0,765 & 0,194 & 0,074 \\
7. Me asusta perder el tiempo y no alcanzar lo que deseo (-) & 0,755 & 0,067 & 0,125 \\
8. Me inquieta tener una vida sin significado y sin trascendencia (-) & 0,700 & $-0,082$ & 0,128 \\
9. Me parece que los trabajos de mis compañeros son mejores que los míos (-) & 0,269 & 0,292 & 0,030 \\
10. Me siento satisfecho (a) conmigo mismo & 0,363 & 0,110 & 0,622 \\
11. Reconozco que soy hábil para muchas cosas & $-0,084$ & 0,277 & 0,450 \\
12. Siento satisfacción con la vida que estoy llevando & 0,293 & $-0,022$ & 0,756 \\
13. Soy una persona a la que le gusta caminar, leer o hacer otras actividades & $-0,125$ & 0,522 & 0,336 \\
recreativas & & & \\
14. Tengo la constancia suficiente para lograr mis metas & $-0,016$ & 0,614 & 0,166 \\
\hline
\end{tabular}

Método de extracción: Análisis de componentes principales. Método de rotación: Normalización Varimax con Kaiser. a La rotación ha convergido en 7 iteraciones.

Tabla 7. Matriz lógica de la escala de motivación de logro para el estudio.

\begin{tabular}{|c|c|c|c|}
\hline Variable & Dimensiones & Indicadores & Reactivos \\
\hline $\begin{array}{l}\text { Motivación de } \\
\text { logro para el } \\
\text { estudio } \\
\text { (Weiner, 1989) } \\
\text { Proceso } \\
\text { de valorar } \\
\text { las propias } \\
\text { creencias en } \\
\text { relación al éxito }\end{array}$ & $\begin{array}{l}\text { Localización } \\
\text { Localización interna } \\
\text { en oposición a la } \\
\text { atribución externa: } \\
\text { Las causas de los } \\
\text { acontecimientos } \\
\text { se interpretan } \\
\text { preferentemente } \\
\text { como causas } \\
\text { internas. }\end{array}$ & $\begin{array}{l}\text { 5. Afección ante la desaprobación } \\
\text { 6. Deseo de tener futuro exitoso } \\
\text { 7. Emoción al tiempo y los deseos } \\
\text { 8. Vida significativa y trascendente }\end{array}$ & $\begin{array}{l}\text { 5. Me afecta que las personas me desaprueben (-) } \\
\text { 6. Me angustia la idea de no tener un futuro exitoso(-) } \\
\text { 7. Me asusta perder el tiempo y no alcanzar lo que deseo (-) } \\
\text { 8. Me inquieta tener una vida sin significado y sin } \\
\text { trascendencia (-) }\end{array}$ \\
\hline $\begin{array}{l}\text { y fracaso de sus } \\
\text { conductas, y de } \\
\text { su disposición } \\
\text { a realizar o } \\
\text { no esfuerzos } \\
\text { para conseguir } \\
\text { beneficios que } \\
\text { dependen más } \\
\text { de las creencias } \\
\text { que de la } \\
\text { realidad tal } \\
\text { como el sujeto la } \\
\text { valora. }\end{array}$ & $\begin{array}{l}\text { Estabilidad } \\
\text { Factores estables vs } \\
\text { factores inestables: } \\
\text { Los factores } \\
\text { estables no } \\
\text { cambiaran porque } \\
\text { no dependen de } \\
\text { nosotros, y que nos } \\
\text { influyen positiva o } \\
\text { negativamente }\end{array}$ & $\begin{array}{l}\text { 1. Firmeza en la toma de decisiones } \\
\text { 2. Sentirse ante los demás } \\
\text { 4. Control de sí mismo } \\
\text { 9. Valoración de los trabajos propios } \\
\text { 13. Hacer actividades normales } \\
\text { 14. Constancia en el logro de metas } \\
\text { 3. Autoevaluación de cualidades } \\
\text { 10. Satisfacción consigo mismo } \\
\text { 11. reconocer habilidades propias } \\
\text { 12. Satisfacción con la vida que lleva }\end{array}$ & $\begin{array}{l}\text { 1. Cada vez que tomo una decisión me mantengo firme en } \\
\text { ella. } \\
\text { 2. Con frecuencia me siento menos que los demás (-) } \\
\text { 4. En general tengo buen control sobre mí mismo } \\
\text { 9. Me parece que los trabajos de mis compañeros son } \\
\text { mejores que los míos (-) } \\
\text { 13. Soy una persona a la que le gusta caminar, leer o hacer } \\
\text { otras actividades recreativas } \\
\text { 14. Tengo la constancia suficiente para lograr mis metas } \\
\text { 3. Creo que tengo más cualidades positivas que negativas } \\
\text { 10. Me siento satisfecho (a) conmigo mismo } \\
\text { 11. Reconozco que soy hábil para muchas cosas } \\
\text { 12. Siento satisfacción con la vida que estoy llevando }\end{array}$ \\
\hline
\end{tabular}




\section{DISCUSIÓN}

Pese a que la escala MLPE no pasó por el juicio de expertos, cuenta con adecuadas propiedades psicométricas de confiabilidad y validez. La escala presenta una buena consistencia interna, ya que el alfa de Crombach excede del criterio mínimo recomendado de 0,70 por Nunnally y Bernstein (1994). Los 14 ítems definitivos presentan $r$ de Pearson Corregida con valores iguales o mayores a 0,20 , lo cual confirma la validez empírica ítem test de la escala.

El análisis factorial con rotación Varimax de la escala y

Tabla 8. Escala para determinar niveles de la MLPE y sus dimensiones.

\begin{tabular}{lcccc}
\hline $\begin{array}{l}\text { Niveles } \\
\text { de MLPE }\end{array}$ & $\begin{array}{c}\text { MLPE } \\
\text { Total }\end{array}$ & $\begin{array}{c}\text { MLPE } \\
\text { Localizacion }\end{array}$ & $\begin{array}{c}\text { MLPE } \\
\text { Control }\end{array}$ & $\begin{array}{c}\text { MLPE } \\
\text { Estabil. }\end{array}$ \\
\hline $\begin{array}{l}\text { Numero de } \\
\text { ítems }\end{array}$ & 14 ítems & 4 ítems & 6 ítems & 4 ítems \\
Promedio & 35,91 & 8,28 & 16.53 & 8,28 \\
Desv. Tip. & 3,565 & 2,173 & 1.376 & 2,173 \\
ALTO & 40 a 42 & 11 a 12 & 18 & 11 a 12 \\
MEDIO & 32 a 39 & 6 a 10 & 15 a 17 & 6 a 10 \\
BAJO & 22 a 31 & 4 a 5 & 6 a 14 & 4 a 5 \\
\hline
\end{tabular}

la consistencia interna de la misma reflejan que los 14 ítems son adecuados indicadores del constructo con cargas factoriales mayores a 0,40 como lo recomienda Field, 2005, (referido por Matos, 2009), excepto el

Tabla 9. Escala para determinar niveles de la MLPE y sus dimensiones.

\begin{tabular}{ccccc}
\hline Percentiles & $\begin{array}{c}\text { MLPE } \\
\text { Localizacion }\end{array}$ & $\begin{array}{c}\text { MLPE } \\
\text { Control }\end{array}$ & $\begin{array}{c}\text { MLPE } \\
\text { Estabilidad }\end{array}$ & $\begin{array}{c}\text { MLPE } \\
\text { Total }\end{array}$ \\
\hline 5 & 5,00 & 14,00 & 9,00 & 29,90 \\
10 & 5,00 & 15,00 & 9,00 & 31,00 \\
15 & 6,00 & 15,00 & 10,00 & 32,00 \\
20 & 6,00 & 15,60 & 10,00 & 33,00 \\
25 & 7,00 & 16,00 & 10,00 & 33,50 \\
30 & 7,00 & 16,00 & 11,00 & 34,00 \\
35 & 7,00 & 16,00 & 11,00 & 35,00 \\
40 & 8,00 & 16,00 & 11,00 & 35,00 \\
45 & 8,00 & 17,00 & 11,00 & 36,00 \\
50 & 8,00 & 17,00 & 12,00 & 36,00 \\
55 & 9,00 & 17,00 & 12,00 & 37,00 \\
60 & 9,00 & 17,00 & 12,00 & 37,00 \\
65 & 9,00 & 17,00 & 12,00 & 38,00 \\
70 & 10,00 & 17,00 & 12,00 & 38,00 \\
75 & 10,00 & 18,00 & 12,00 & 38,00 \\
80 & 10,00 & 18,00 & 12,00 & 39,00 \\
85 & 11,00 & 18,00 & 12,00 & 40,00 \\
90 & 11,00 & 18,00 & 12,00 & 40,00 \\
95 & 12,00 & 18,00 & 12,00 & 41,00 \\
\hline
\end{tabular}

ítem $9(0,292)$. Un análisis de componentes principales mostró la existencia de tres factores en el cuestionario, agrupados por un número proporcional de ítems por cada factor y que responde a las definiciones operacionales de las dimensiones de Localización, Control y Estabilidad.

Para un uso interno se construyeron escalas y percentiles a fin de ubicar cualitativamente la motivación para el estudio de los alumnos. Pese a que las dimensiones Control y Estabilidad tienen distribución de datos hacia la derecha, es decir la mayoría contesto favorablemente, se construyó la escala de tres niveles: Alto, Medio Y Bajo y se mantuvo toda vez que será utilizado solo para uso institucional. De esto queda para más adelante incluir reactivos en sentido negativo a la variable e introducir reactivos que midan el grado de sinceridad, dada la naturaleza de la evaluación, es decir se trata de exámenes de ingreso a la universidad.

Como una contribución a la temática, hemos querido aportar un instrumento útil de evaluación de la motivación para el estudio. Se precisan procedimientos y las variables cruciales del problema, para que resulten verdaderamente útiles para ser aplicados en el proceso educativo de los ingresantes a la universidad, o bien para actuar acertadamente en las sesiones de aprendizaje en las aulas universitarias y lograr el buen rendimiento académico de los alumnos. El presente trabajo contribuye, según creemos, a enriquecer el enfoque subjetivo de la medición mediante autoinforme por vía virtual, aportando una escala fácilmente aplicable que posee las propiedades psicométricas necesarias. Con esta medida se evalúa, como lo plantea B. Weiner (1989), la percepción subjetiva que tienen las personas sobre la motivación de logro para el estudio, que inician sus estudios universitarios. El estudio psicométrico indica que el cuestionario reproduce fielmente la estructura del modelo teórico propuesto.

\section{REFERENCIAS BIBLIOGRÁFICAS}

Clark-Carter, D. (2002).Investigación cuantitativa en psicología. México D.F: Oxford

Decy y Ryan (1987). The support of autonomy and the control of behavior, Journal of Personality and Social Psychology, 53, 1024-1037.

Kramp, U (2008). Equivalencia entre los modelos de análisis factorial de los ítems y teoría de respuesta a los ítems en la evaluación de las propiedades psicométricas de los instrumentos de medición psicológica. Revista Peruana de Psicometría. 1 (1), julio-diciembre.

Matos, L. (2009). Adaptación de dos cuestionarios de motivación: Autorregulación del Aprendizaje y 
Clima de Aprendizaje. Persona. 12, pp. 167-185. Nunnally, J. C. y Bernstein, I. H. (1994). Psychometric theory. (3rd Ed.). Nueva York: McGraw-Hill.

Núñez, J.L, Martin-Albo, J. y Navarro, J.G, (2005). Validación de la versión española de la Échele Motivation en Education. Psicothema. 17, 002, 344-349
Weiner, B. y Ghaman, G. (1989). Understanding the Motivational Role of Affect: Live-span research from an Attributional Perspectiva. Cognition and Emotion, 3 (4), 401-419). 